EOMmUn: Communication et organisation

Or

La communication, dimension oubliée de l'intelligence économique

\title{
Entrevue avec Christian Harbulot
}

Nicolas Moinet

\section{OpenEdition}

Journals

Édition électronique

URL : http://journals.openedition.org/communicationorganisation/3919

DOI : 10.4000/communicationorganisation.3919

ISSN : $1775-3546$

Éditeur

Presses universitaires de Bordeaux

Édition imprimée

Date de publication : 1 décembre 2012

Pagination : 152-159

ISBN : 978-2-86781-772-4

ISSN : $1168-5549$

Référence électronique

Nicolas Moinet, « Entrevue avec Christian Harbulot», Communication et organisation [En ligne],

42 | 2012, mis en ligne le 01 décembre 2013, consulté le 01 mai 2019. URL : http://

journals.openedition.org/communicationorganisation/3919; DOI : 10.4000/

communicationorganisation.3919 


\section{Entrevue avec Christian Harbulot}

Christian Harbulot, 60 ans, est Directeur de l'École de Guerre Économique et directeur associé du cabinet Spin Partners. Expert international en intelligence économique, Christian Harbulot initie des travaux de recherches sur les problématiques d'affrontements économiques et les stratégies de puissance depuis le milieu des années 1980. En 1990, alors qu'il est directeur des relations extérieures de l'ADITECH (future ADIT, société nationale d'intelligence stratégique), il publie Techniques offensives et guerre économique. Ses travaux interpellent Edith Cresson, alors Premier Ministre, et lui valent d'être nommé conseiller personnel d'Henri Martre au Commissariat Général au Plan. Co-auteur (avec Philippe Baumard, Philippe Clerc et Jean-Louis Levet) du rapport qui donnera naissance à l'intelligence économique en France. En 1993, il est recruté par le Groupe Défense Conseil International comme Directeur des opérations de son département d'intelligence économique Intelco. Il y mène à la fois des activités de conceptualisation, de conseil et de sensibilisation à l'IE. Constatant le fort déficit culturel des entreprises françaises en matière d'IE, il crée l'École de Guerre Économique (EGE) en 1997 avec le Général Pichot-Duclos. Depuis le début des années 2000, il concentre ses travaux sur les questions de guerre de l'information. Christian Harbulot enseigne également à l'ESSEC et à l'École des Mines de Paris. Il est aussi conférencier régulier à l'Institut des Hautes Études de la Défense Nationale. Il est membre du Conseil Scientifique de la Formation et de la Recherche Stratégique (CSFRS) et Vice Président de L'Institut International d'Intelligence Économique et Stratégique (Institut de l'IE). Auteur d'une cinquantaine d'articles et de nombreux ouvrages, il a coordonné le Manuel d'intelligence économique paru aux Presses Universitaires de France en 2012.

Dans son $\mathrm{n}^{\circ}$ de décembre 2012 - janvier 2013, le magazine l'Expansion a marqué les esprits avec une couverture qui titrait : « 2013 : L'année de la guerre économique ". Cette notion est aujourd'hui très utilisée par les médias ou les politiques mais peu par les universitaires. Pourquoi ?

Longtemps considérée comme un concept exotique par le monde universitaire, la guerre économique est train de devenir une réalité incontestable des relations internationales. Les penseurs qui stigmatisent les rapports de force entre puissances ${ }^{1}$ ont dû s'incliner devant l'évolution très démonstrative des relations internationales. Aux actes de portée géopolitique (arme du gaz utilisée par la Russie pour renforcer son statut de puissance ou remise en cause de la suprématie monétaire du dollar par

1 B. BADIE, L'impuissance de la puissance, Paris, Fayard, 2004. 
l'Iran par exemple) se sont ajoutés des faits de nature géoéconomique (tensions diplomatiques sur la question des ressources entre la Chine et le Japon, politique protectionniste affichée par les États-Unis à l'encontre de la Chine dans le domaine de l'industrie solaire...). Ces différents faits soulignent l'intérêt d'une grille de lecture des affrontements assimilés à la guerre économique.

\section{Pour vous, la guerre économique serait donc consubstantielle à l'histoire humaine et non une dérive ou un versant négatif du développement ?}

Le début du $\mathrm{XXI}^{\mathrm{e}}$ siècle est marqué par une remise en cause de la vision positive du développement héritée des révolutions industrielles et de la pacification relative découlant de la mondialisation des échanges comme l'ont laissé entendre la plupart des économistes libéraux. Dans le même ordre d'idées, la Pax Americana officialisée par la disparition de l'URSS à l'origine du mythe de la fin de l'histoire ${ }^{2}$ fait place à une multipolarisation des risques d'affrontement, en raison de la limitation progressive des ressources, des tensions croissantes sur la question de l'énergie, des crises structurelles du monde occidental provoquées par la désindustrialisation et de la volonté de conquête commerciale des nouveaux entrants. De facto, nous entamons une longue période de tensions en tout genre dont le suivi ne pourra pas se limiter à un discours lénifiant sur la recherche de croissance. Étudier la guerre économique ${ }^{3}$ implique de passer du non dit au dit, ce qui est un exercice difficile compte tenu de la volonté quasi universelle des belligérants de masquer la nature de leurs affrontements non militaires. Les travaux menés depuis seize ans sous ma direction au sein de l'École de Guerre Économique de Paris nous ont permis de jeter les bases de cette grille de lecture indispensable pour décrypter les stratégies d'accroissement de puissance par l'économie et les rapports de force qu'elles génèrent.

Peut-on dès lors aller jusqu'à dire que la guerre économique a été dissimulée? De l'antiquité à l'ère des révolutions industrielles, la prédominance de la réalité économique est une constante dans la nature des rapports de force entre les individus, les groupes et les États. Le professeur Edward Mead Earl de l'Institute for Advanced Study a souligné le rapport dialectique entre la dimension politique et économique de la puissance ${ }^{4}$ :

«S'il était possible de séparer la puissance économique de la puissance politique, ce ne serait que dans les sociétés les plus primitives. Dans les temps modernes (avec l'émergence de l'État national, l'expansion de la civilisation européenne dans le monde entier, la révolution industrielle, et les progrès constants de la technologie militaire) il nous a fallu envisager la question de l'interdépendance

2 F. FUKUYAMA, La fin de l'bistoire et le dernier homme, Paris, Flammarion, 1992.

3 C. HARBULOT, Comment travailler sur l'absence d'bistoire, chronique du 7 novembre 2012, www. lesinfluences.fr.

4 E. MEAD EARL, Les Maîtres de la Stratégie, Tome 1, Paris, Flammarion, 1986. 
entre, d'une part, la force commerciale, financière et industrielle et d'autre part, la force politique et militaire. Cette corrélation est l'un des problèmes les plus critiques de l'art de gouverner. Elle implique la sécurité d'une nation et, dans une large mesure, de déterminer le degré de vie, de liberté, de propriété, de bonheur dont peut jouir l'individu ».

Il en est de même pour la théorie réaliste des relations internationales qui, même si elle a négligé l'aspect économique de la recherche de puissance, décrit 1 ' « animus dominandi » comme l'élément constitutif de l'ensemble des associations humaines, de toutes les relations sociales, et donc de la vie politique à la fois interne et internationale. Hans Morgenthau ${ }^{5}$ souligne que la politique internationale est une lutte pour la puissance. Or la puissance n'est pas que militaire. Mais contrairement à la guerre militaire, la guerre économique n'est pas devenue un objet de débat dans les milieux politiques et académiques. Et la dissimulation de la guerre économique se vérifie aussi bien dans les stratégies de domination mises au point par les empires coloniaux que dans les stratégies de rattrapage des pays qui voulaient éviter la colonisation ou qui furent par la suite en recherche de puissance.

\section{Comment analysez-vous cette dissimulation de la guerre économique dans le cadre des stratégies de domination puis de rattrapage ?}

La question religieuse contribua à masquer la finalité des affrontements comportant une part non négligeable de bénéfices économiques. Après avoir longtemps été masquée par le prétexte de l'évangélisation des peuples considérés comme primitifs, la dissimulation de la guerre économique résulte d'une nouvelle phase de construction de la puissance à l'aube des révolutions industrielles. Si la guerre militaire a évolué en fonction des inventions techniques, la notion de puissance subit une véritable métamorphose sous l'impact de la création des empires économiques. $\mathrm{La}$ naissance du libéralisme met en perspective un nouveau mode d'accroissement de puissance par la conquête commerciale qui devient une alternative au mode traditionnel de conquête territoriale.

La conquête commerciale peut déboucher sur la guerre commerciale qui devient un moyen de coercition lorsque les pays visés par les commerçants britanniques s'opposent à leur volonté de pénétrer leurs marchés intérieurs. Pour imposer leurs produits sur les marchés du Moyen-Orient et de l'Asie orientale, les Britanniques instaurèrent la pratique de la « politique de la canonnière ». Celle-ci connut son apogée lors du blocus du port d'Alexandrie par la Royal Navy en 1840-1841 puis lors des deux guerres de l'opium qui ont successivement opposé la Chine à la Grande Bretagne, puis la Chine à une coalition de pays occidentaux. Le monde occidental impose alors à un pays théoriquement indépendant le commerce de la drogue

5 H. MORGENTHAU, Politics Among Nations. The struggle for Power and Peace, New York, Mac Graw-Hill, 1948, p. 29. 
par des moyens militaires. William Jardine qui dirigeait la firme d'opium Jardine\&Matheson à Hong Kong, légitima cette action en se faisant le chantre de la "libre entreprise, indépendante et sans contrainte ${ }^{6}$ ".

Les guerres de l'opium donnent une telle lisibilité de l'agressivité économique qu'elles amènent des pays comme le Japon ${ }^{7}$ à refondre l'identité du pays par une politique de puissance fondée sur l'expansion économique, symbolisée par le slogan "Un pays riche, une armée forte ». L'arrivée au pouvoir de l'Empereur Mutsuhito, en 1868, est ainsi le début d'une série de réformes dont l'objectif est de rattraper le retard face à l'Occident. Le Japon mit plus d'un siècle à élaborer les fondements d'une économie au service de la puissance. À la fin des années 1980, la Central Intelligence Agency publie le rapport Japan $2000^{8}$ rédigé par groupe de travail composé par des personnalités du monde civil et militaire. Ce document est une rare trace écrite contemporaine d'un texte de portée gouvernementale sur les rapports de force économique entre deux puissances. La partie la plus démonstrative du texte porte sur la dénonciation de la " propagande japonaise » pour masquer les mesures protectionnistes que ce pays applique à l'égard des autres économies de marché et son non respect du libéralisme économique. Certains passages du rapport stigmatisaient en ces termes la stratégie de puissance japonaise : "Les membres du club du Chrysanthème (qui réunit l'élite des milieux politiques et industriels du Japon) pensent que le système occidental est condamné à disparaître et ils agissent dans toute la mesure de leur possible pour en hâter la fin ». La stratégie du rattrapage du Japon menée depuis l'ère Meiji lui permit de se hisser au deuxième rang de l'économie mondiale au bout d'un peu plus d'un siècle d'effort. À la fin des années 1980, des voix s'élevèrent aux Etats-Unis et en Europe dans les milieux politiques et économiques pour dénoncer l'expansionnisme nippon et le recours à des techniques de guerre économique.

L'agressivité commerciale de l'ex-empire du Soleil Levant à l'égard de l'Occident fut stoppée sous l'impulsion des autorités américaines par différents moyens (attaques répétées pour casser le protectionnisme invisible nippon, déstabilisation de son système bancaire par le refus d'accorder un serpent monétaire lors de la crise financière asiatique, blocage de sa stratégie sur le technoglobalisme9, limitation des jeux d'influence japonais dans le système politico administratif américain). La

6 B. BRIZAY, Le sac du palais d'Eté. Seconde guerre de l'opium, Paris, éditions du Rocher, 2011.

7 P.-F. SOUYRI, La nouvelle histoire du Japon, Paris, éditions Perrin, 2010.

8 Le rapport Japan 2000, qui fut très vite retiré de la circulation à la suite de la protestation des autorités japonaises, annonçait une tension dans les négociations entre les États-Unis et le Japon sur l'ouverture de son marché intérieur et l'accès à l'actionnariat de ses grandes entreprises.

9 Elaboré en 1987 par le Ministère de l'Industrie et du Commerce japonais (MITI), le technoglobalisme visait à éviter un parasitage de la recherche par les pratiques concurrentielles et aussi à combler l'écart entre le Nord et le Sud en créant un patrimoine commun de l'humanité. Il faisait suite à un durcissement américain dans le domaine des brevets et des échanges scientifiques avec le Japon. 
chute du mur de Berlin priva le Japon de l'objet de son chantage implicite à l'égard de l'Amérique. Les autorités gouvernementales américaines n'avaient plus à craindre le basculement de cet allié/ancien adversaire dans la sphère d'influence soviétique, au cas où les États-Unis n'accorderaient pas à Tokyo une marge de manœuvre significative dans la construction de la puissance économique nipponne. Le coup d'arrêt brutal donné à la puissance économique japonaise n'invalida pas pour autant la pertinence des stratégies de rattrapage.

\section{Justement, compte tenu de l'émergence de nouvelles puissances, comment se caractérisent les stratégies de rattrapage au XXI siècle ?}

Les stratégies de rattrapage s'articulent autour d'objectifs élémentaires fortement dépendant de leur contexte géographique et culturel. Pour le cas du Japon, son insularité l'a conduit à se doter en priorité d'une infrastructure à vocation maritime (chantiers navals, ports), puis dans un second temps de bâtir les fondements d'une économie industrielle. Plusieurs pays comme la Corée du Sud, l'Inde, le Brésil, la Chine suivirent par la suite son exemple sans pour autant copier son modèle. La Corée du Sud imita la démarche japonaise en privilégiant la construction navale et la formation de grands conglomérats industriels privés, les chaebols, qui sont les équivalents coréens des keiretsu japonais, anciens zaibatsu dissous par les autorités d'occupation américaines après la défaite du Japon en 1945. L'Inde a emprunté d'autres chemins pour se positionner comme acteur dominant dans l'industrie informatique mondiale. Le Brésil a développé des stratégies de rattrapage en misant sur le secteur énergétique (le pétrole non conventionnel par les gisements off-shore et ses réserves amazoniennes, l'eau par le nombre de barrages hydrauliques, les énergies renouvelables). L'État brésilien ${ }^{10}$ a ainsi fait de la firme Petrobras la tête de pont de sa stratégie d'influence géoéconomique. En matière de soft power ${ }^{11}$, le Brésil peaufine aussi son image de nouvelle puissance du développement durable en revendiquant également le fait d'être l'un des pays les plus propres du monde par sa production électrique.

Quant à la Chine, elle a construit sa stratégie de rattrapage en jouant sur l'ouverture (création de zones économiques spéciales et politique d'attractivité très forte afin de favoriser l'investissement étranger), contrairement au Japon qui chercha à capter la connaissance tout en fermant l'accès à son marché intérieur. Le point commun entre les deux pays est la priorité donnée dans leurs phases de développement mutuel à la

10 N. MAZZUCCHI, «L'énergie, source de la nouvelle puissance brésilienne », Nouvelle Revue Géopolitique, $\mathrm{n}^{\circ}$ 3, Paris, 2012.

11 Action indirecte qui vise à mettre une puissance en rapport de force favorable sur un sujet de débat de portée internationale. Les stratégies de soft power recoupent aussi les stratégies d'influence destinées à amener des pays cibles à s'aligner sur les prises de position d'une puissance conformément à ses intérêts. 
conquête des marchés extérieurs. Dans les deux cas de figure, cette forme d'agressivité commerciale a abouti à des réactions hostiles de la part des États-Unis et déclenché des débats médiatiques sur la problématique de la guerre économique ${ }^{12}$ dans le monde occidental. La Chine est accusée notamment de mener une stratégie de noyautage des organismes de normalisation pour imposer ses normes ${ }^{13}$.

\section{Mais dans un monde globalisé, ces stratégies de rattrapage ne vont-elles pas céder nécessairement le pas à une pacification des échanges?}

Rien n'est moins sûr car plusieurs facteurs risquent de faire resurgir les problématiques d'affrontement et de domination :

- l'acquisition des ressources énergétiques et minières ;

- les enjeux territoriaux liés à leur localisation géographique ;

- les problèmes de dépendance économique ;

- les nouvelles formes de colonisation culturelle par la société de l'information ;

- les possibilités de renversements d'alliance.

Il existe désormais un déséquilibre entre les dynamiques de puissance des nouveaux entrants et la manière dont le monde occidental s'est habitué à gérer sa puissance économique sans rivaux véritables. Les nouveaux entrants ont comme priorité la conquête des marchés extérieurs pour financer leur politique d'accroissement de puissance alors que les pays du monde occidental ont dissocié la problématique de puissance (principalement militaire et diplomatique) des logiques de guerre économique passées sous silence depuis le milieu du XIX ${ }^{\mathrm{e}}$ siècle. La politique de déréglementation initiée dans le monde occidental accentue ce paradoxe. Les champions nationaux sont démantelés en Europe alors que les nouveaux entrants bâtissent leur compétitivité en misant sur le potentiel de consortiums financés par des banques contrôlées directement ou indirectement par le pouvoir politique du pays. Ce type de fonctionnement est incompatible avec le système compétitif du monde occidental. Il en découle un déséquilibre concurrentiel qui affaiblit les pays industrialisés qui ont découplé la question de l'accroissement de puissance de la problématique de la compétition économique. Un tel déséquilibre est renforcé par l'importance prise par la finance dans le fonctionnement de l'économie de marché occidentale. Les marchés financiers influent sur la définition des enjeux stratégiques dans la mesure où les politiques ont pris l'habitude de substituer le court terme aux critères temporels de construction et de préservation de la puissance.

12 Dossier « 2013, l'année de la guerre économique », L’Expansion, n 780, décembre 2012, Paris. 13 Ibid., p. 44. 